\title{
Comparison of the Fourier-Kirchhoff, Pennes and DPL thermal models of a single layer tissue
}

\author{
by M. Strąkowska*, G. De Mey** and B. Więcek*
}

* Lodz University of Technology, Institute of Electronics, 211/215 Wólczańska St. 90-924 Łódź, Poland, maria.strakowska@p.lodz.pl

${ }^{\star \star}$ Gent University, Department of Electronics and Information Systems, Sint-Pietersnieuwstraat 41, B-9000 Gent, Belgium

\section{Abstract}

This paper deals with comparison of Fourier - Kirchhoff (FK), Pennes and Dual Phase Lag (DPL) heat transfer 1D models of a single-layer tissue. Thermal modelling presented in this paper is based on the concept of thermal impedance by using the Laplace transform for $s=j \omega$. In such a case, the models for simple geometry can be solved analytically. Then, the poles of the thermal impedance are identified using e.g. Vector Fitting method which allows calculating the thermal impedance as a sum of partial fractions. It corresponds directly to the Foster Network of a thermal object. Models are compared both in frequency and time domain for wide range of frequencies.

\section{Introduction}

Thermal modelling of the skin is the field of interest for many researches. Knowing both thermal parameters of the skin and its reaction for thermal excitation is useful to describe pathologies of the skin tissue like a tumours, psoriasis and inflammations including pre-cancerous lesions and cancers. Skin is a complex structure with three main layers. There are many approaches to thermal modelling such structure. The most common is the Fourier - Kirchhoff heat transfer model. Many models are the modification on the Fourier - Kirchhoff one, e.g. the Pennes [1]model which assumes the presence of perfusion. The other models are SPL and DPL - single-phase-lag and dual-phase-lag ones. It assumes that there is relaxation time $\tau_{q}$ which indicates the time lag caused by the finite propagation time of heat flux in a tissue. This model was first described by Cattaneo [2] and Vernotte [3] in 1958. In 1995 Tzou [4] proposed DPL (dual-phase-lag) model which generalized previous approach and assumes another time constant $\tau_{\mathrm{T}}$ which is known as the thermalization time. In the literature there are many simulation results of these models. Most of them present the numerical solutions. The value of parameters especially for DPL model are differ in many papers [5-14]. The thermalization time vary from few miliseconds $[11,12]$ to few tens seconds $[13,14]$. The same for the relaxation time. In this paper the comparison of F-K, Pennes and DPL model is introduced by analysing them analytically in the frequency domain.

\section{Thermal models of a tissue in frequency domain}

\subsection{The Fourier - Kirchhoff model}

According the Fourier- Kirchhoff law, heat is transferred in between 2 points in the space if there is a thermal gradient $[15,16]$.

$$
q=-k \frac{\partial T}{\partial x}
$$

where $q$ is the heat flux in $\mathrm{W} / \mathrm{m}^{2}$ and $k$ is the thermal conductivity.

The well-known Fourier - Kirchhoff law is expressed as equation (2)

$$
k \frac{\partial^{2} T}{\partial x^{2}}-C_{t h} \frac{\partial T}{\partial x}=-q_{v}
$$

where $q_{v}$ is power density dissipated in the tissue in $\mathrm{W} / \mathrm{m}^{3}, \mathrm{C}_{\text {th }}$ is thermal capacity in $\mathrm{J} / \mathrm{Km}^{3}$.

In order to simplify the calculus, one can present equation (2) in the frequency domain by using the Laplace transform for $s=j \omega$.

where $L$ is diffusion length and is equal to:

$$
\nabla^{2} T-\frac{T}{L^{2}}=-\frac{q_{v}}{k}
$$

$$
L=\sqrt{\frac{k}{j \omega C_{t h}}}
$$

The differential equation $\mathrm{x}$ has its analytical solution for 1D geometry in frequency domain:

$$
T(j \omega)=A e^{-\frac{d}{L}}+B e^{\frac{d}{L}}+\frac{q_{v}}{j \omega C_{t h}}
$$

where $A$ and $B$ are the integration constants, that can be found using the boundary conditions. 


\subsection{The Pennes model}

The Pennes model of heat transfer assumes that the perfusion $\mathrm{w}$ generates a=or/and absorbs an additional heat in a tissue [1]. In addition, the model can apply a metabolic heat generation in a tissue. The perfusion coefficient simple modifies the differential equation of $\mathrm{F}-\mathrm{K}$ heat transfer.

The heat conduction model is now presented as:

$$
k \nabla^{2} \mathrm{~T}-C_{t h} \frac{\partial T}{\partial t}-\mathrm{w} C_{t h b}=-\left(q_{v}+q_{v m}\right)
$$

where the thermal capacity of blood $C_{t h b}=q_{b} C_{b}$ can differ from the thermal capacity of a tissue $C_{t h}$, and $\rho_{b}$ and $c_{b}$ denote the density and the specific heat of blood.

The perfusion coefficient w modifies the diffusion length $L$.

$$
L(j \omega)=\sqrt{\frac{k}{j \omega C_{t h}+w C_{t h b}}}
$$

At last, the analytical solution of the model can be presented as

$$
T(j \omega)=A e^{-\frac{d}{L}}+B e^{\frac{d}{L}}+\frac{q_{v}+q_{v m}}{j \omega C_{t h}}
$$

\subsection{Dual Phase Lag model}

The Dual Phase Lag model assumes that the heat is transferred not only due to the temperature gradient. In addition, thermal energy can be generated and transfers if both the temperature and the heat flux are varying in time. In consequence, 2 additional thermal time constants are introduced displaying both an additional relaxation of heat $\tau_{q}$ and a lag of temperature $\tau[4,17,18]$.

$$
q+\tau_{q} \frac{\partial q}{\partial t}=-k\left(\frac{\partial T}{\partial x}+\tau_{T} \frac{\partial^{2} T}{\partial x \partial t}\right)
$$

Using the Laplace transform for the equation (9), it is possible to redefine the heat flux and thermal conductivity in the more general forms:

where

$$
q=-\tilde{k} \frac{\partial T}{\partial x}
$$

$$
\tilde{k}=\tilde{k} \frac{1+j \omega \tau_{T}}{1+j \omega \tau_{q}}
$$

Introducing the new thermal conductivity (11), one can solve the heat transfer equation in a tissue in the frequency domain using the classical heat transfer equation (2)

\subsection{Boundary conditions and analitycal solution of the models for a single layer tissue}

Let us assume that the tissue has the thickness $d$ and is heating by the flux $q_{0}$ at the frontal side (for $x=0$ ). This case corresponds to the well-known cold stress examination practiced in medicine. First, the skin tissue is cooled down and then it is warming by flowing blood and convection from ambient. This process can be approximated by the heating using the external heat flux. At the bottom side $(x=d)$, either isothermal or forced convection boundary condition can be assumed due to the high blood flow. The blood has the body-core temperature $T_{b}$.

$$
q_{0}=-\left.\tilde{k} \frac{\partial T}{\partial x}\right|_{x=0}
$$

Using (12) for isothermal boundary condition at the bottom side of a tissue, it is possible to calculate integration constants $\mathrm{A}$ and $\mathrm{B}$ by solving the set of 2 linear equations.

$$
\begin{gathered}
A-B=-\frac{q_{0} L}{k} \\
A e^{-\frac{d}{L}+B e^{\frac{d}{L}}}=T_{b}-\frac{q_{v}+q_{v m}}{j \omega C_{t h}}
\end{gathered}
$$
in the form:

Alternatively, it is possible to define the convective boundary condition at the bottom side of the sample for $x=d$

$$
A\left(\frac{\tilde{k}}{L} e^{-\frac{d}{L}}-h e^{-\frac{d}{L}}\right)+B\left(-\frac{\tilde{k}}{L} e^{\frac{d}{L}}-h e^{\frac{d}{L}}\right)=h \frac{q_{v}+q_{v m}}{j \omega C_{t h}}
$$

Analytical solution of equations (13) and (14) leads to determine both temperature and thermal impedance in frequency domain that can be presented graphically as the Nyquist's plot.

$$
T(x-=0, j \omega)=A+B+\frac{q_{v}+q_{m}}{j \omega C_{t h}}
$$




$$
T(x=d, j \omega)=A e^{-\frac{d}{L}}+B e^{\frac{d}{L}}+\frac{q_{v}+q_{v m}}{j \omega C_{t h}}
$$

\section{Methods and simulation results}

Using the initial and boundary conditions for single tissue layer, the models can easily be solved in frequency domain. Then the Laplace transform has to be calculated for power $P(t)$, and finally, the thermal impedance is estimated as:

$$
Z_{t h}(j \omega)=\frac{T(j \omega)}{P(j \omega)}
$$

where power is typically the scaled Heaviside's step function with its Laplace transform $P(j \omega)=P_{0} / j \omega$.

Then Vector Fitting method is applied in order to approximate thermal impedance as the sum of partial fractions. It leads to the distribution of thermal time constants and finally thermal response in time.

$$
T(t) \approx \sum_{i=1}^{N} R_{i} P_{0}\left(1-e^{\frac{t}{\tau_{i}}}\right)
$$

In order to compare 3 presented thermal models, the simulations were performed for a single layer tissue sample with the parameters presented in Table 1.

Table 1. The model parameters

\begin{tabular}{|l|l|l|}
\hline Parameter & Unit & Simulation 1 \\
\hline Thermal conductivity $\mathrm{k}$ & $\mathrm{W} /(\mathrm{m} \cdot \mathrm{K})$ & 1 \\
\hline thickness of the sample d & $\mathrm{m}$ & 0.001 \\
\hline thermal capacity of a tissue $C_{\text {th }}$ & $\mathrm{J} / \mathrm{m}^{3} \mathrm{~K}$ & $3.96 \cdot 10^{6}$ \\
\hline thermal capacity of blood $C_{\text {thb }}$ & $\mathrm{J} / \mathrm{m}^{3} \mathrm{~K}$ & $4.16 \cdot 10^{6}$ \\
\hline perfusion of a tissue w & $1 / \mathrm{s}$ & 0.00125 \\
\hline relaxation time constant of the DPL $\tau_{q}$ & $\mathrm{~S}$ & $10^{-3}$ \\
\hline Inertial time constant of the DPL model $\tau_{T}$ & $\mathrm{~s}$ & $\tau T=10^{-2}$ \\
\hline
\end{tabular}

The analysis were performed for angular frequency in the wide range starting at $\omega_{\min }=10^{-6} 1 / \mathrm{s}$ up to $\omega_{\max }=10^{6}$ $1 / \mathrm{s}$. The rational approximation of the thermal impedance in the frequency domain was calculated for the $7^{\text {th }}$ order polynomial in the denominator. In consequence, 7-pole approximation of thermal impedance was used $\left(n_{p}=7\right)$. The calculations were performed for $N=10000$ angular frequencies not uniformly distributed in the chosen range $\left(\omega_{\min }, \omega_{\max }\right)$.

The results both in time and frequency domains are presented in graphical form in Figures 1 and 2.
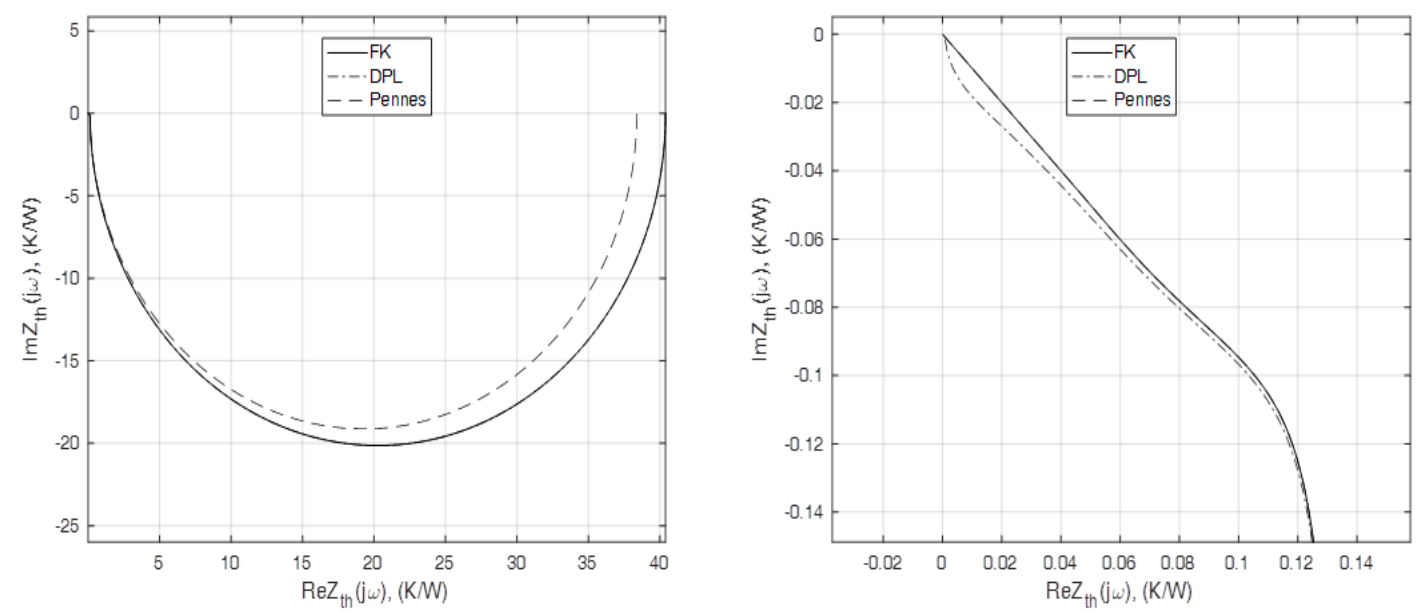

Fig 1. Thermal impedance of the slab for FK, DPL and Pennes heat transfer models for $\omega \in\left(10^{-6}, 10^{6}\right) 1 / \mathrm{s}$, at the frontal side of the sample $(x=0)$ 

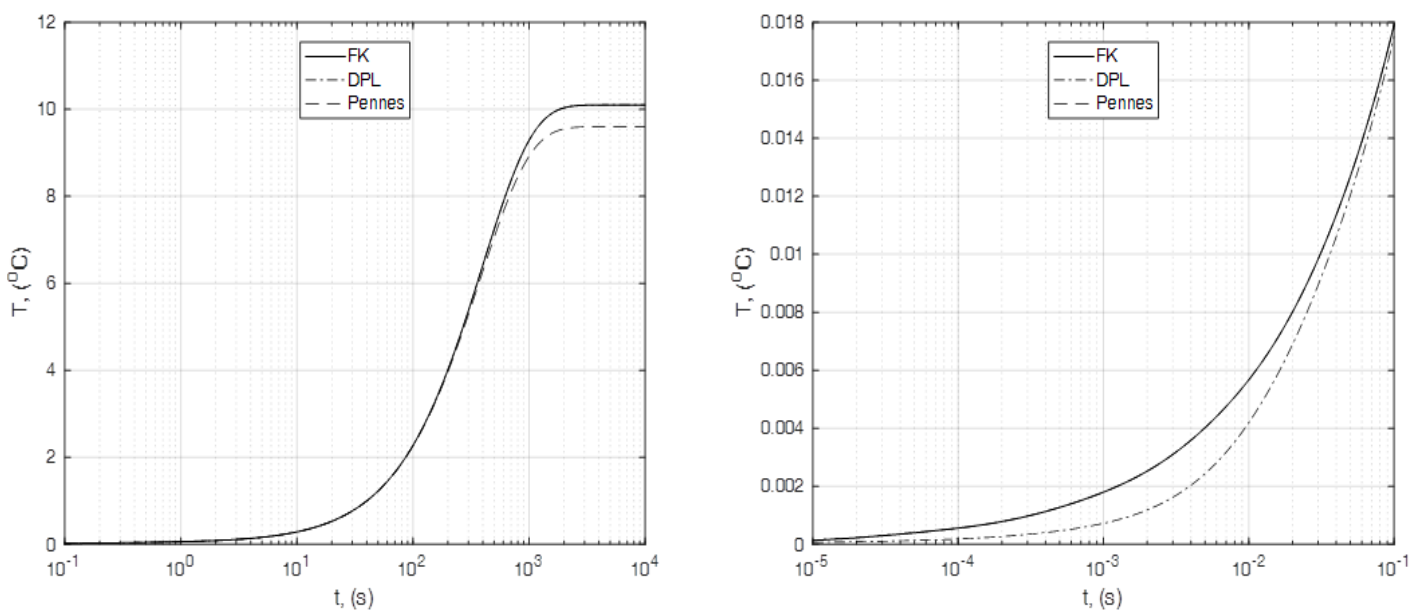

Fig 2. Temperature rise at the front site of the tissue $(x=0)$ : for the transient and steady states and at the beginning of thermal process

The complex thermal impedance in the wide range of the angular frequencies is presented in Figure 1. It is possible to notice, that for low angular frequencies, the thermal impedance obtained from F-K and DPL models are overlapping.

In the case of the high angular frequency range, the thermal impedance obtained from $\mathrm{F}-\mathrm{K}$ and Pennes models are overlapping. The curves approach the origin of the coordinate system with the angle of $45^{\circ}$. For high frequencies, the thermal impedance for DPL model significantly differs from F-K and Pennes models exhibiting the higher phase lag.

For transient and steady states, temperature evolutions for F-K and DPL models are overlapping, in contrast to the beginning of heating where temperature rises for F-K and Pennes models are the same. As expected, at the beginning of thermal process there is the significant temperature lag for the DPL model - Figure 2.

\section{Conclusion}

The analysis of three thermal models of skin tissue are presented in this paper (Fourier- Kirchhoff, Pennes and DPL). Models are solved analytically in frequency domain. The approximation using Vector Fitting method was done in order to fit the model to the sum of exponential function. The comparison of the models is done for low and high frequencies. There are big deviation of the parameters of relaxation and temperature lag for DPL model for tissues in literature. To confirm investigation the experiment has to be evaluated. Future work will be focused on introduce these models for experimental data.

\section{REFERENCES}

[1] Pennes H., Analysis of tissue and arterial blood temperature in resting human forearm, Journal Appl.Physiol., Vol. 1, 1948, pp. 93-122.

[2] Cattaneo C., A form of heat conduction equation which eliminates the paradox of instantaneous propagation, Compte Rendus, 247, 1958, 431-433.

[3] Vernotte P., Les paradoxes de la theorie continue de l'equation de la chaleur, Compte Rendus, 246, 1958, 31543155.

[4] Tzou D.Y., Macro-to Microscale Heat Transfer: The Lagging Behavior, 2nd ed., Wiley, 2014, ISBN: 978-1-11881822-0.

[5] Zhang Y., Generalized dual-phase lag bioheat equations based on nonequilibrium heat transfer in living biological tissues, International Journal of Heat and Mass Transfer, Vol. 52, 2009, pp. 4829-4834.

[6] Zubert M., Raszkowski T., Samson A., Zajac P. Methodology of determining the applicability range of the DPL model to heat transfer in modern integrated circuits comprised of FinFETs, Microelectronics Reliability, vol. 91, 2018, 139-153.

[7] Dinesh Kumar, Surjan Singh, Neha Sharma, K.N. Rai, Verified non-linear DPL model with experimental data for analyzing heat transfer in tissue during thermal therapy, International Journal of Thermal Sciences, 133, 2018, 320-329.

[8] Saeed T., Abbas I. Finite element analyses of nonlinear DPL bioheat model in spherical tissues using experimental data, Mechanics Based Design of Structures and Machines, 2020, DOI: 10.1080/15397734.2020.1749068. 
[9] Sunil Kumar Sharma and Dinesh Kumar, A Study on Non-Linear DPL Model for Describing Heat Transfer in Skin Tissue during Hyperthermia Treatment, Entropy 2020, 22, 481.

[10] Hooshmand P., Moradi A., Khezry B., Bioheat transfer analysis of biological tissues induced by laser Irradiation, International Journal of Thermal Sciences, 90, 2015, 214-223.

[11] Tareq Saeed \& Ibrahim Abbas (2020): Finite element analyses of nonlinear DPL bioheat model in spherical tissues using experimental data, Mechanics Based Design of Structures and Machines, DOI: $10.1080 / 15397734.2020 .1749068$

[12] P. Hooshmand, A. Moradi, B. Khezry, Bioheat transfer analysis of biological tissues induced by laser Irradiation, International Journal of Thermal Sciences, 90, 2015, 214-223.

[13] Dinesh Kumar, Surjan Singh, Neha Sharma, K.N. Rai, Verified non-linear DPL model with experimental data for analyzing heat transfer in tissue during thermal therapy, International Journal of Thermal Sciences, 133, 2018, 320-329.

[14] Sunil Kumar Sharma, and Dinesh Kumar, A Study on Non-Linear DPL Model for Describing Heat Transfer in Skin Tissue during Hyperthermia Treatment, Entropy 2020, 22, 481.

[15] Bejan A., Heat transfer. Wiley, New York, 1993.

[16] Incropera F. and De Witt D., Introduction to heat transfer. Wiley, New York, 1985.

[17] Strakowska M., Strakowski R., Strzelecki M., De Mey G., Wiecek B., Thermal modelling and screening method for skin pathologies using active thermography, Biocybernetics and Biomedical Engineering, 10.1016/j.bbe.2018.03.009. vol. 38, no. 3, 2018, pp. 602-610.

[18] Strakowska M., Chatzipanagiotou P., De Mey G., Chatziathanasiou V., Wiecek B., Novel software for medical and technical Thermal Object Identification (TOI) using dynamic temperature measurements by fast IR cameras, 14th Quantitative InfraRed Thermography Conference, QIRT 2018, June 25-29, 2018, Berlin. 